\title{
Metabolomics profiling in plasma samples from glioma patients correlates with tumor phenotypes
}

\author{
Hua Zhao ${ }^{1}$, Amy B. Heimberger ${ }^{2}$, Zhimin Lu ${ }^{3}$, Xifeng Wu ${ }^{1}$, Tiffany R. Hodges ${ }^{2}$, Renduo \\ Song ${ }^{1}$, Jie Shen ${ }^{1}$ \\ ${ }^{1}$ Department of Epidemiology, The University of Texas MD Anderson Cancer Center, Houston, TX 77030, USA \\ ${ }^{2}$ Division of Neuro-Surgery, The University of Texas MD Anderson Cancer Center, Houston, TX 77030, USA \\ ${ }^{3}$ Department of Neuro-Oncology, The University of Texas MD Anderson Cancer Center, Houston, TX 77030, USA \\ Correspondence to: Hua Zhao, e-mail: hzhao2@mdanderson.org
}

Keywords: metabolomics, glioma, tumor phenotype

Received: September 17, 2015

Accepted: February 13, 2016

Published: March 07, 2016

\section{ABSTRACT}

Background: Tumor-based molecular biomarkers have redefined in the classification gliomas. However, the association of systemic metabolomics with glioma phenotype has not been explored yet.

Methods: In this study, we conducted two-step (discovery and validation) metabolomic profiling in plasma samples from 87 glioma patients. The metabolomics data were tested for correlation with glioma grade (high vs low), glioblastoma (GBM) versus malignant gliomas, and IDH mutation status.

Results: Five metabolites, namely uracil, arginine, lactate, cystamine, and ornithine, significantly differed between high- and low-grade glioma patients in both the discovery and validation cohorts. When the discovery and validation cohorts were combined, we identified 29 significant metabolites with 18 remaining significant after adjusting for multiple comparisons. Those 18 significant metabolites separated highfrom low-grade glioma patients with $91.1 \%$ accuracy. In the pathway analysis, a total of 18 significantly metabolic pathways were identified. Similarly, we identified 2 and 6 metabolites that significantly differed between GBM and non-GBM, and IDH mutation positive and negative patients after multiple comparison adjusting. Those 6 significant metabolites separated IDH1 mutation positive from negative glioma patients with $94.4 \%$ accuracy. Three pathways were identified to be associated with IDH mutation status. Within arginine and proline metabolism, levels of intermediate metabolites in creatine pathway were all significantly lower in IDH mutation positive than in negative patients, suggesting an increased activity of creatine pathway in IDH mutation positive tumors.

Conclusion: Our findings identified metabolites and metabolic pathways that differentiated tumor phenotypes. These may be useful as host biomarker candidates to further help glioma molecular classification.

\section{INTRODUCTION}

During the past decade, glioma classification has moved from histopathology only to molecular characterization. Allelic loss of $1 \mathrm{p} / 19 \mathrm{q}$ and $p 53$ gene mutation has been deemed as the hallmarks for low grade oligodendroglioma and astrocytomas, respectively $[1,2]$. The results from The Cancer Genome Atlas (TCGA) Research Network and several other studies have pinpointed phosphoinositide 3-kinase (PI3K), RTK/RAS/
PI3K, EGF receptors (EGFR), p53, retinoblastoma (RB), and PTEN signaling alterations as driving forces for highgrade glioma tumorigenesis $[3,4]$.

The renewed interest of Warburg effect has drawn attention to the understanding of how underlying metabolic alterations may contribute to the aggressive phenotype in tumors $[5,6]$. Although the data are still limited, promise has already been shown of using metabolomics in characterizing gliomas [7]. For example, utilizing metabolomic profiling in 69 Grade II to IV glioma tumor 
tissues, Chinnaiyan et al. identified a metabolic classifier that could group glioma tumors into 3 different subclasses with distinct prognostic relevance [7]. Metabolomic platforms quantify small-molecule metabolites in biospecimens and can be used to evaluate the role of metabolic alterations in chronic disease. Because it takes into account genetic regulation, altered kinetic activity of enzymes, genomics and proteomics, metabolomics reflects changes in phenotype, and thereby function [8,9]. Studies using metabolomics in various cancers have shown that there are common alterations in metabolism in patients with cancer, but there are also disease specific alterations in metabolism [10-14].

It has recently become clear that altered metabolic homeostasis plays important roles in carcinogenesis. Recent results from limited clinical and epidemiological studies have suggested that metabolic disorders may affect the progression of high grade gliomas. For example, Derr et al. reported that high grade gliomas patients with hyperglycemia have a shortened overall survival [15]. Chambless et al. observed that pre-existing diabetes and elevated body mass index (BMI) are independent risk factors for high grade glioma progression [16]. However, to our knowledge, there have been no studies to date examining the role of small-molecule metabolites in the circulation in relation to glioma characterization. In the current study, utilizing targeted metabolomics analysis, we analyzed 224 known metabolites from 25 key metabolic pathways in plasma samples from 87 glioma patients. We hypothesized that plasma metabolite profiles could differentiate glioma tumor phenotypes.

\section{RESULTS}

Basic demographic characteristics of the patient cohort were shown in Table 1. Briefly, the mean age was 45 years old, and nearly $60 \%$ of the study subjects were male. The majority of study subjects were Caucasians $(86.2 \%)$. About $20 \%$ of the study subjects used steroid during the treatment. Seizure medication use was prevalent $(72.4 \%)$. In addition, $44.8 \%$ of the study subjects had dyslipidemia diagnosis.

Targeted metabolic profiling was performed using LC-QQQ-MS on a total of 87 plasma samples from both the discovery and validation cohorts. The profiling was performed in two phases, discovery $(N=42)$ and validation $(N=45)$. From a targeted 224 metabolites, a total of 157 metabolites were detected in both discovery and validation cohorts. Following log transformation and imputation with minimum observed values for each metabolite, we first attempted to identify metabolites that differed significantly between high- and low-grade gliomas. In the discovery cohort, 8 plasma metabolites differed significantly between high- and low-grade gliomas. They were listed in Table 2. Among them, 5 plasma metabolites were increased in high-grade gliomas; whereas 3 were decreased. The top two significant metabolites were uridine $(P=0.004)$ and ornithine $(P=0.016)$. Compared to low-grade gliomas, levels of plasma uridine were 2.27-fold elevated in high grade gliomas. However, after adjusting multiple comparisons, none of the metabolites was significant ( $q$ value $\leq 0.05$ ). In the validation cohort, we identified 10 metabolites that differed significantly between high- and low-grade gliomas. They were listed in Table 2. Among them, levels of 6 plasma metabolites were increased in high-grade gliomas and 4 were decreased. The top three significant metabolites elevated in high-grade gliomas were uracil $(P=0.007)$, arginine $(P=0.008)$, and pyroglutamic acid $(P=0.016)$. Levels of plasma uracil were 2.19-fold increased in high-grade gliomas relative to low-grade gliomas. However, after adjusting for multiple comparisons, none of the metabolites was significant. In comparison, of the top 10 significant metabolites identified from the discovery and validation cohorts, 5 significant metabolites $(P \leq 0.05)$ identified in the discovery cohort were also significant in the validation cohort. They were uracil, arginine, lactate, cystamine, and ornithine. Next, we conducted a meta-analysis by combining the samples from discovery and validation cohorts. The heatmap was shown in Supplemental Materials. A total of 29 significant metabolites were identified and top 10 are listed in Table 2. These top 5 metabolites were uridine $\left(P=6.43 \times 10^{-6}, q=0.001\right)$, uracil $\left(P=1.87 \times 10^{-5}, q=0.001\right)$, arginine $\left(P=1.07 \times 10^{-4}\right.$, $q=0.005)$, agmanite $\left(P=1.05 \times 10^{-4}, q=0.005\right)$, and ornithine $\left(P=2.68 \times 10^{-4}, q=0.005\right)$ (Figure 1A). Levels of plasma uridine were 4.86-fold higher in high- than in low-grade gliomas. On the other hand, levels of plasma arginine were 4.02-fold lower in high- than in low-grade gliomas. After adjusting multiple comparisons, there were still 18 significant metabolites left. In further PLS-DA analysis using those 18 significant metabolites, glioma patients could be successfully classified into high- and low-grade groups with $91.1 \%$ of accuracy (Figure 2A).

We next investigated whether plasma metabolites could differentiate GBM patients versus malignant glioma patients. Due to the small sample size of GBM, we combined discovery and validation cohorts together ( $n=18: 4$ in discovery and 14 in validation). Two metabolites were identified that differed significantly between GBM and malignant glioma patients based on both $P$ and $q$ values. They were uridine $\left(P=3.76 \times 10^{-4}\right.$, $q=0.015)$ and ornithine $\left(P=9.36 \times 10^{-4}, q=0.038\right)$ (Figure 1B). Then, we explored whether plasma metabolites could differentiate IDH mutation status. Altogether, we had 36 patients whose IDH mutation status was known. Among them, 12 were negative and 24 were positive. The heatmap was shown in Supplemental Materials. Six metabolites were identified that significantly differed between IDH mutation positive and negative tumors based on both $P$ and $q$ values (Table 3 ). The top 2 metabolites were N-acetylputrescine 
Table 1: Demographic characteristics of the patient cohort

\begin{tabular}{l}
\hline Age, mean (range) \\
\begin{tabular}{|l|c|}
\hline Gender (\%) & $45(22-72)$ \\
\hline Male & $50(57.5)$ \\
\hline Female & $37(42.5)$ \\
\hline Ethnicity (\%) & $75(86.2)$ \\
\hline Caucasian & $10(11.5)$ \\
\hline Others & $2(2.3)$ \\
\hline Unknown & $92(60-100)$ \\
\hline KPS, mean (range) & $18(20.7)$ \\
\hline Steroid use (\%) & $69(79.3)$ \\
\hline Yes & \\
\hline No & $63(72.4)$ \\
\hline Seizure Medication Use (\%) & $24(27.6)$ \\
\hline Yes & \\
\hline No & $22(25.3)$ \\
\hline Dyslipidemia during study (\%) & $4(4.6)$ \\
\hline Yes & $61(70.1)$ \\
\hline No & \\
\hline Test not done & $39(44.8)$ \\
\hline Dyslipidemia diagnosis (\%) & $48(55.2)$ \\
\hline Yes & $5(5.7)$ \\
\hline No & \\
\hline Antiglycemic medication Use & \\
\hline Yes & \\
\hline No & \\
\hline
\end{tabular}
\end{tabular}

$\left(P=9.12 \times 10^{-4}, q=0.036\right)$ and trimethylamine-N-oxide (TMAO) $(P=0.006, q=0.043)$ (Figure 1C). Levels of plasma $\mathrm{N}$-acetylputrescine were 2.96-fold lower in $I D H$ mutation positive relative to the negative glioma patients. On the other hand, levels of plasma Methionine were 2.08fold higher in $I D H$ mutation positive than negative glioma patients. In further PLS-DA analysis using those 6 significant metabolites, glioma patients could be successfully classified by $I D H-1$ status with $94.4 \%$ of accuracy (Figure $2 \mathrm{~B}$ ).

Next, we conducted a pathway analysis which integrates an enrichment analysis and pathway topology analysis. Pathway topology analysis is used to analyze the impact of one metabolite or a group of metabolites in a certain pathway. A total of 18 metabolic pathways were significantly differed between high- and low-grade glioma patients based on a combination of $P$ and $q$ value (Table 4). Although D-arginine/D-ornithine metabolism and pyrimidine metabolism were the two most significant pathways, the metabolites involved in both pathways have very low impact factors (0 and 0.09 , respectively), suggesting they are unlikely to be the metabolic pathways contributing to the difference between high- and low-grade gliomas. On the other hand, arginine/proline metabolism, another significant metabolic pathway $(P=0.002$ and $q=0.010)$, had high impact factor $(0.55)$. A total of 16 out of 77 metabolites possibly involved in arginine and proline metabolism were analyzed in this study. Among them, 4 differed significantly between high- and low-grade gliomas $(P \leq 0.05)$. They were arginine $\left(P=1.07 \times 10^{-4}\right)$, fumaric acid $(P=0.049)$, agmetine $(P=0.007)$, and ornithine $\left(P=2.68 \times 10^{-4}\right)$. Particularly, arginine and ornithine are key metabolites in the pathway and play critical roles.

Using a similar approach, we also studied whether metabolic pathways could differentiate GBM from malignant glioma patients, and $I D H$ mutation status. For GBM differentiation, we didn't observe any significant metabolic pathways associated with GBM based on $P$ and $q$ value. However, we did identify metabolic pathways that significantly differed by $I D H$ mutation status (Table 4) such as, tryptophan metabolism, D-arginine and D-ornithine metabolism, and arginine and proline metabolism pathways. When further examined for impact factor, only the arginine and proline metabolism pathway had a high impact factor (0.541). As shown in Figure 3, in arginine 
Table 2: List of top 10 metabolites in discovery, validation, and combined cohorts*

\begin{tabular}{|c|c|c|c|c|c|c|c|c|c|c|c|}
\hline \multicolumn{4}{|c|}{ Discovery $(n=42)$} & \multicolumn{4}{|c|}{ Validation $(n=47)$} & \multicolumn{4}{|c|}{ Combined meta-analysis $(n=89)$} \\
\hline Metabolite & $P$ value & $\begin{array}{c}\text { Fold } \\
\text { change }\end{array}$ & FDR & Metabolite & $P$ value & $\begin{array}{c}\text { Fold } \\
\text { change }\end{array}$ & FDR & Metabolites & $P$ value & $\begin{array}{c}\text { Fold } \\
\text { change }\end{array}$ & FDR \\
\hline Uridine & 0.004 & 2.27 & 0.542 & Uracil & 0.007 & 2.19 & 0.301 & Uridine & $6.43 \mathrm{E}-06$ & 4.86 & 0.001 \\
\hline Ornithine & 0.016 & 1.83 & 0.542 & Arginine & 0.008 & 2.12 & 0.301 & Uracil & $1.87 \mathrm{E}-05$ & 4.32 & 0.001 \\
\hline Cystamine & 0.019 & 1.80 & 0.542 & $\begin{array}{l}\text { Pyroglutamic } \\
\text { Acid }\end{array}$ & 0.016 & 1.92 & 0.301 & Arginine & $1.07 \mathrm{E}-04$ & 4.02 & 0.005 \\
\hline Glucosamine & 0.020 & 1.70 & 0.542 & Lactate & 0.028 & 1.66 & 0.301 & Agmanite & $1.05 \mathrm{E}-04$ & 3.78 & 0.005 \\
\hline Uracil & 0.020 & 1.73 & 0.542 & Cystamine & 0.037 & 1.52 & 0.301 & Ornithine & $2.68 \mathrm{E}-04$ & 3.46 & 0.005 \\
\hline Arginine & 0.025 & 1.66 & 0.542 & Ornithine & 0.038 & 1.50 & 0.301 & Biotin & $3.54 \mathrm{E}-04$ & 3.24 & 0.005 \\
\hline Biotin & 0.028 & 1.53 & 0.542 & Xanthurenate & 0.040 & 1.50 & 0.305 & Lactate & $4.27 \mathrm{E}-04$ & 3.08 & 0.007 \\
\hline \multirow[t]{3}{*}{ Lactate } & 0.039 & 1.42 & 0.599 & Oxalic Acid & 0.042 & 1.42 & 0.334 & Cystamine & $9.46 \mathrm{E}-04$ & 3.09 & 0.024 \\
\hline & & & & Agmanite & 0.046 & 1.45 & 0.334 & Glucosamine & 0.004 & 2.67 & 0.024 \\
\hline & & & & Niacinamide & 0.047 & 1.33 & 0.378 & Oxalic Acid & 0.002 & 2.04 & 0.036 \\
\hline
\end{tabular}

*Metabolites whose fold changes in red reflect an increase accumulation in high grade glioma cases and metabolites whose fold changes in blue reflect a decrease accumulation in high grade glioma cases. The analysis was adjusted by age, gender, ethnicity, KPS, steroid use, seizure medication use, and dyslipidemia diagnosis.

Uridine

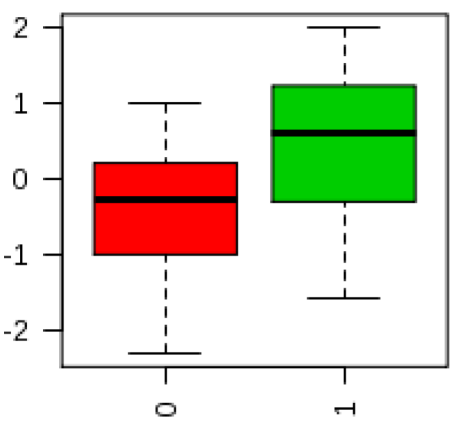

Uracil

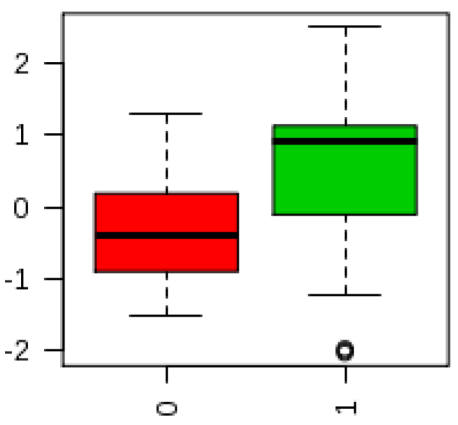

A
Uridine

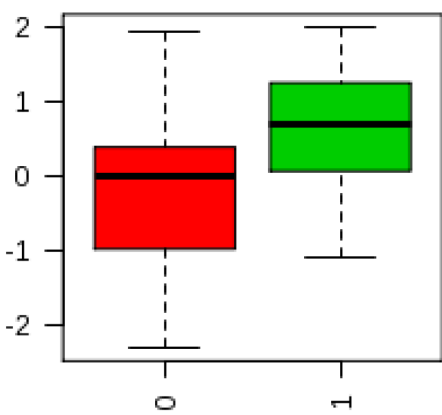

Ornithine

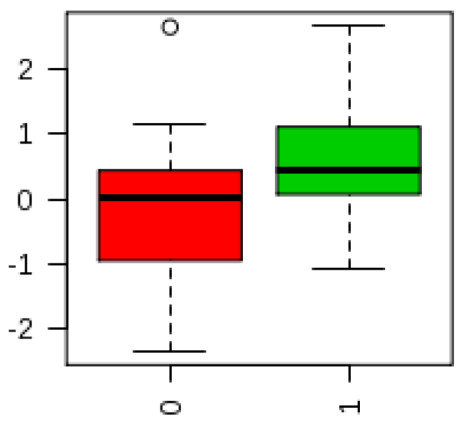

B

\section{N-Acetylputrescine}

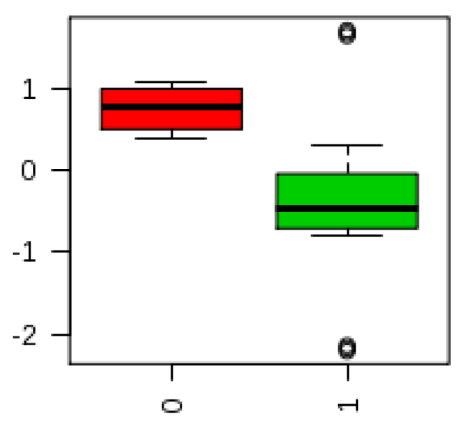

Trimethylamine-N-oxide (TMAO)

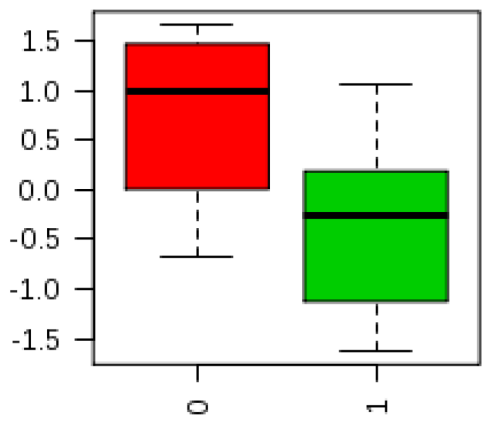

C

Figure 1: Box plot of top 2 metabolites that significantly differed between tumor characteristics. (A) High and low grade (1 vs 0); (B) GBM and malignant gliomas (1 vs 0); (C) IDH mutation positive and negative status (1 vs 0). 
Table 3: Significant metabolites differentiating IDH mutation status

\begin{tabular}{|l|c|c|c|}
\hline \multicolumn{1}{c}{ Metabolite } & P value & Fold change & FDR \\
\hline N-acetylputrescine & $9.12 \mathrm{E}-04$ & 2.96 & 0.036 \\
\hline $\begin{array}{l}\text { trimethylamine-N-oxide } \\
\text { (TMAO) }\end{array}$ & 0.006 & 2.37 & 0.043 \\
\hline Nicotinate (Niacin) & 0.009 & 2.19 & 0.043 \\
\hline Arginine & 0.009 & 2.52 & 0.043 \\
\hline Glucosamine & 0.013 & 1.96 & 0.047 \\
\hline Methionine & 0.016 & 2.08 & 0.047 \\
\hline
\end{tabular}

* Metabolites whose fold changes in red reflect an increase accumulation in $I D H$ mutation positive cases and metabolites whose fold changes in blue reflect a decrease accumulation in IDH mutation positive cases. The analysis was adjusted by age, gender, ethnicity, KPS, steroid use, seizure medication use, and dyslipidemia diagnosis.

and proline metabolism pathway, 4 out of 16 analyzed metabolites had $P$ value less than 0.05 (labeled in red). Two metabolites had $P$ value ranging from 0.05 to 0.10 (labeled in green), and another 2 had $P$ value ranging from 0.10 to 0.20 (labeled in blue). Notably, levels of intermediate metabolites in the creatine pathway such as guanidoacetic acid, creatine, and reatinine were all significantly lower in $I D H$ positive than negative patients. At the same time, levels of sarcosine, the downstream metabolite in the pathway, were higher in $I D H$ positive than negative patients.

\section{DISCUSSION}

In a recent publication by Eckel-Passow et al., gliomas could be classified into five principal groups on the basis of three tumor markers, including chromosome 1p/19q co-deletion, IDH mutation, and TERT promoter mutations in tumors [17]. In another study, low-grade gliomas could be further classified into three molecular groups based on $I D H, 1 p / 19 q$, and TP53 status [4]. Those studies show the promise of utilizing molecular markers for glioma classification. However, none of the existing studies have looked at the contribution from metabolic homeostasis and how individual difference in metabolic profiles in circulation may correlate with glioma phenotypes. In current study, we used plasma metabolomics profiling to assess the differences between high- and low-grade, GBM and malignant glioma, and IDH positive and negative gliomas. Compared to tumor tissues, plasma is a metabolite-rich matrix, readily available via minimal invasive samples techniques, and thereby, is highly amenable to global metabolic profiling studies in gliomas.

In comparison of glioma grade, we identified a total of 29 metabolites in plasma samples that could

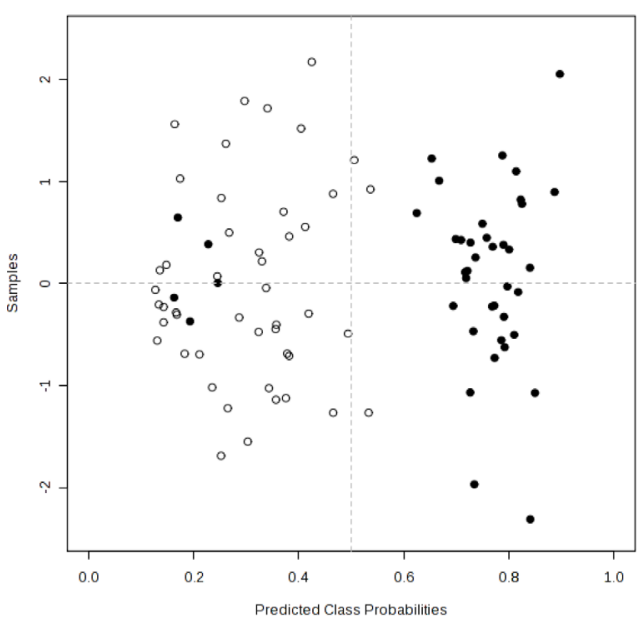

A

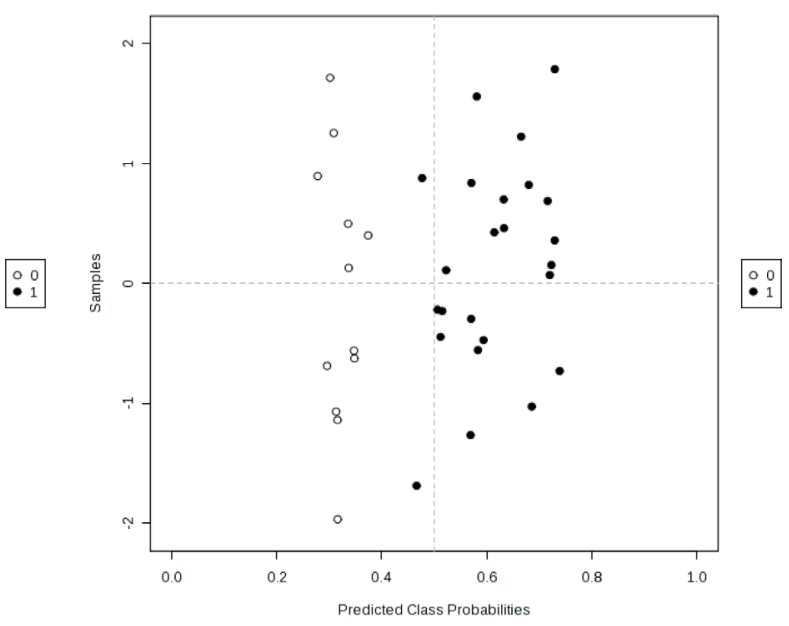

B

Figure 2: PLS-DA analysis to differentiate tumor grade and $I D H$ mutation status using significant metabolites. (A) High vs low grade (1 vs 0); (B) $I D H$ positive vs negative (1 vs 0 ). 
Table 4: Identification of significant metabolic pathways associated with grade and IDH mutation status

\begin{tabular}{|c|c|c|c|c|}
\hline Pathway Name & $P$ value & Fold change & FDR & Impact \\
\hline \multicolumn{5}{|c|}{ High vs low grade } \\
\hline D-Arginine and D-ornithine metabolism & $1.44 \mathrm{E}-06$ & 13.45 & $8.19 \mathrm{E}-05$ & 0 \\
\hline Pyrimidine metabolism & $1.45 \mathrm{E}-05$ & 11.14 & $4.14 \mathrm{E}-04$ & 0.091 \\
\hline Pantothenate and CoA biosynthesis & $8.67 \mathrm{E}-05$ & 9.35 & 0.002 & 0.180 \\
\hline Glutathione metabolism & $1.29 \mathrm{E}-04$ & 8.96 & 0.002 & 0.002 \\
\hline beta-Alanine metabolism & $7.18 \mathrm{E}-04$ & 7.24 & 0.008 & 0.011 \\
\hline Glycolysis or Gluconeogenesis & $9.34 \mathrm{E}-04$ & 6.98 & 0.009 & 0.199 \\
\hline Biotin metabolism & 0.001 & 6.73 & 0.01 & 0.203 \\
\hline Tryptophan metabolism & 0.001 & 6.5 & 0.01 & 0.102 \\
\hline Arginine and proline metabolism & 0.002 & 6.47 & 0.01 & 0.553 \\
\hline Amino sugar and nucleotide sugar metabolism & 0.002 & 6.37 & 0.01 & 0.091 \\
\hline Pyruvate metabolism & 0.003 & 5.67 & 0.018 & 0.32 \\
\hline Propanoate metabolism & 0.007 & 4.93 & 0.034 & 0.002 \\
\hline Phenylalanine metabolism & 0.011 & 4.54 & 0.047 & 0.198 \\
\hline Glyoxylate and dicarboxylate metabolism & 0.012 & 4.46 & 0.048 & 0.162 \\
\hline Tyrosine metabolism & 0.013 & 4.36 & 0.049 & 0.162 \\
\hline Taurine and hypotaurine metabolism & 0.014 & 4.28 & 0.049 & 0.353 \\
\hline Nicotinate and nicotinamide metabolism & 0.015 & 4.20 & 0.049 & 0.135 \\
\hline Methane metabolism & 0.016 & 4.16 & 0.049 & 0.018 \\
\hline
\end{tabular}

IDH mutation status (positive vs negative)

\begin{tabular}{|l|c|c|c|c|}
\hline Tryptophan metabolism & $5.12 \mathrm{E}-04$ & 7.58 & 0.019 & 0.102 \\
\hline D-Arginine and D-ornithine metabolism & $6.70 \mathrm{E}-04$ & 7.31 & 0.019 & 0 \\
\hline Arginine and proline metabolism & 0.002 & 6.07 & 0.043 & 0.541 \\
\hline
\end{tabular}

significantly differentiate high- from low-grade glioma patients. Our results suggest significant changes in pathways related to nucleotides (e.g. pyramidine), amino acids (e.g. arginine, glutathione, alanine, tryptophan), and carbohydrate (e.g. glycolysis and pyruvate) metabolism. This is consistent with the profile of tumor metabolism, which needs to acquire key nutrients from circulation for rapid ATP generation, increased biosynthesis of macromolecules, and maintenance of appropriate cellular redox status [18]. One of the most consistent metabolites observed in this study is arginine, whose plasma levels were significantly lower in high-grade than in low-grade glioma patients in discovery, validation, and combined cohorts. Arginine is a semi-essential amino acid in humans. It has been established that an increased dependence on exogenous arginine is typical for many malignant tumor cells both in vitro and in vivo [19-20]. Also, a growing number of tumors are being identified as deficient in the arginine anabolic enzyme argininosuccinate synthetase (ASS) and, thus auxotrophic for arginine [21, 22]. Pavlyk et al. showed that arginine deprivation could affect glioblastoma cell adhesion, invasiveness and actin cytoskeleton organization [23]. Thus, the observed difference in plasma arginine levels between high- and low-grade glioma patients could be due to the difference in arginine dependence. Another consistent metabolite is lactate, whose plasma levels were significantly higher in high-grade cases relative to low-grade cases. This is expected since lactate is one of the main metabolic "waste" or by-products during tumor metabolism. Lactate can actually be transported back to the liver to produce glucose (known as the Cori cycle), which imposes an energy burden on whole-body metabolism [24]. Increased flux through this pathway has been postulated to be one of the drivers of energy dissipation in cachexia, which is a contributing factor in one third of all cancer deaths.

$I D H$ mutation is not restricted to a specific histopathological type of glioma but instead was associated with a distinctive tumor-cell metabolism [25]. However, how metabolomics profiles in plasma may be differentiated by $I D H$ mutation status is unknown. In the current study, we identified 6 plasma metabolites that differed significantly by $I D H$ mutation status. The most significant metabolite is $\mathrm{N}$-acetylputrescine. $\mathrm{N}$-Acetylputrescine is the most abundant of all polyamines both in normal individuals and in patients with leukemia 
[26]. Currently, the role of plasma N-acetylputrescine may play in glioma tumorigenesis is unclear. In the pathway analysis, we found tryptophan metabolism, D-arginine and D-ornithine metabolism, and arginine and proline metabolism were significantly different based on $I D H$ mutation status. Within the arginine and proline metabolism pathway, we found levels of intermediate metabolites in the creatine pathway, from guanidoacetic acid, creatine, and creatinine, were all significantly lower in $I D H$ mutation positive than in negative patients. However, levels of sarcosine, the downstream metabolite in the pathway, were higher in $I D H$ mutation positive than in negative patients. Creatine is usually detected in magnetic resonance imaging research of brain tumors. The creatine levels reflect energy buffering and transport. Our results may suggest an increased activity of creatine pathway in $I D H$ mutation positive patients, which is in line with the findings that creatine tended to be low in the highgrade tumors [27]. Among Grade II to IV glioma cases, IDH positive status is associated with improved survival [17]. Thus, although survival data are not available in this study yet because of short follow-up time, the plasma metabolites that significantly differed by $I D H$ mutation status may potentially have prognostic relevance. Clearly, further investigation of plasma metabolites and survival in glioma patients will be our next step.

Mutated IDH protein leads to the generation of excessive amount of the metabolite 2-hydrocyglutarate (2-HG) in glioma tumor cells [28]. Since 2-HG is a small molecule it seems possible that it could reach the systemic circulation and that elevated 2-HG plasma levels may help to identify patients harboring $I D H$ mutated gliomas. In the current study, 2-HG metabolite was detectable. However, no significant association was observed between plasma 2-HG levels and $I D H$ mutation status $(P=0.458)$ or tumor grade $(P=0.743)$. In fact, our observation is consistent with the reported from Capper et al. [29]. In their study of 16 glioma patients, no correlation was observed between serum $2-H G$ levels and IDH1/2 status or tumor size. Thus, 2-HG in circulation may not be a useful marker for glioma molecular classification.

One limitation for circulation metabolomics analysis is the vagueness of the source of the metabolites. In the case of gliomas, tumor cells, tumor infiltrating immune cells and different types of stroma cells all produce metabolites and some of those metabolites could be released and thereby enter the circulation. Currently, there are active studies underway performing metabolic profiling in various types of cancer related cells, including cancer stem cells [30], tumor infiltrating immune cells [31], and other stroma [32-34], in attempt to elucidate their metabolic profiles to dissect the sources of the circulating metabolic factors. The knowledge gained from those studies can help us better interpret the results from this study and provide guidance for circulating biomarker discovery.

In summary, our study showed distinct signatures of plasma metabolite levels by glioma grade (high vs low)

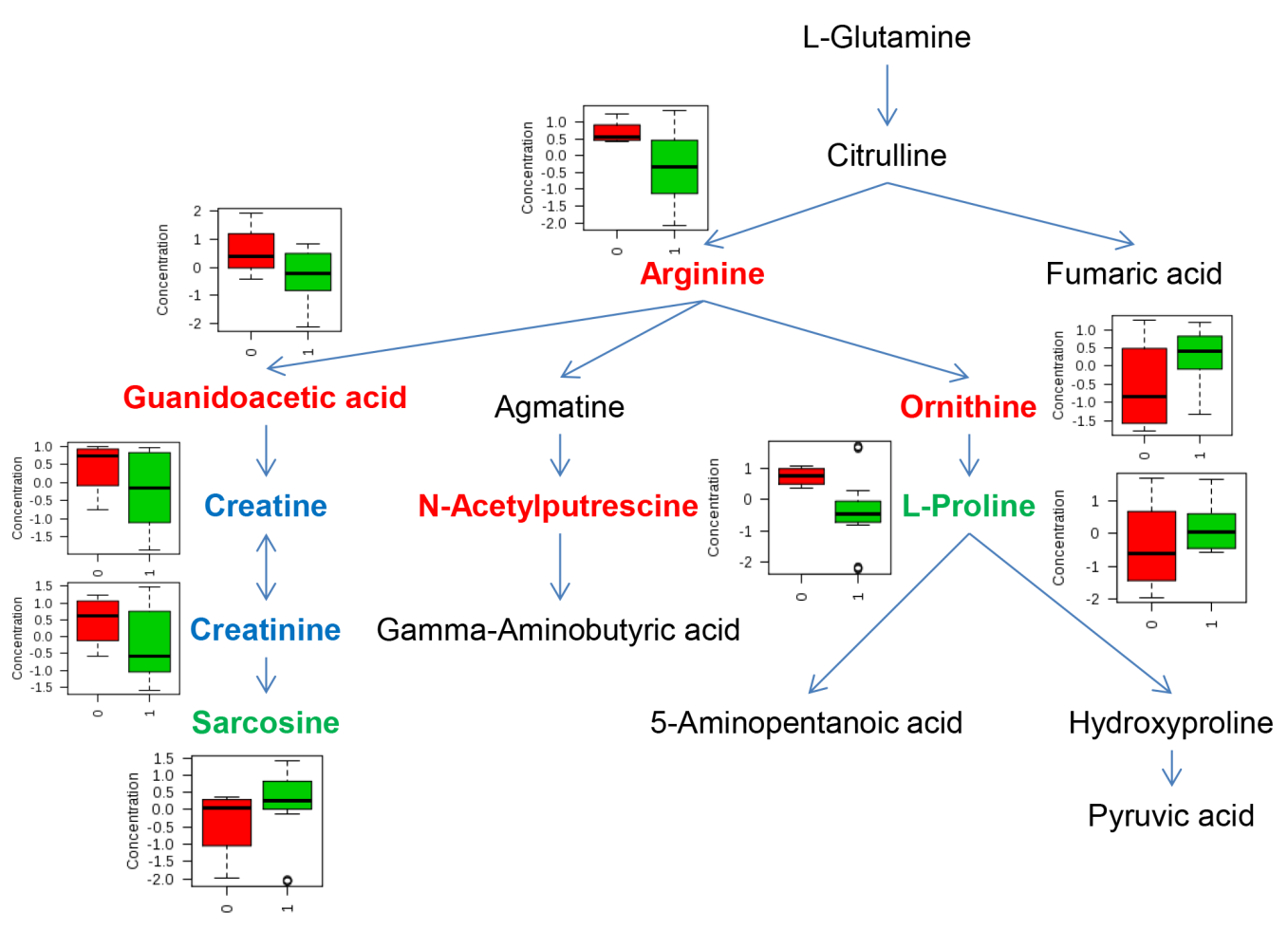

Figure 3: Metabolites involved in arginine and proline pathway that significantly differed by $I D H$ mutation status. Metabolites in red reflect $P$ value $\leq 0.05$, metabolites in blue reflect $P$ value between 0.05 to 0.10 , and metabolites in green reflect $P$ value between 0.10 to 0.20 . In box-plot, red indicates $I D H$ mutation negative and green indicates $I D H$ mutation positive. 
and $I D H$ mutation status. Additional weaknesses include small sample size, retrospective study design, incomplete exposure information, and the lack of matched tumor/ adjacent normal tissues. Nevertheless, our study provides the first evidence to support the role of circulating metabolites in glioma phenotypes. Such knowledge, if confirmed, could help stratify glioma patients and enable personalize medicine.

\section{MATERIALS AND METHODS}

\section{Study subjects}

Case patients were recruited from The University of Texas M. D. Anderson Cancer Center (Houston, TX). All patients with either newly diagnosed or previously treated, histopathologically confirmed glioma who were registered at the Cancer Center between April 2014 and July 2015 were eligible for our study. The exclusion criteria for case patients were currently undergoing chemotherapy or radiation therapy, prior cancer (except for non-melanoma skin cancer), and any blood transfusion in the 6 months prior to recruitment. Written informed consent was obtained from each study subject. A total of 87 glioma patients were included in the study. We divided the study subjects into two cohorts, discovery and validation. The discovery cohort included 42 glioma patients, including 25 low-grade glioma (Grade I: $n=5$; Grade II: $n=20$ ) and 17 high-grade glioma patients (Grade III: $n=13$; Grade IV GBM: $n=4)$. The validation cohort had 45 glioma patients, including 17 low-grade glioma (Grade I: $n=4$; Grade II: $n=13$ ) and 28 high-grade glioma patients (Grade III: $n=14$; Grade IV GBM: $n=14$ ). The demographic and clinical data for the cases were obtained from medical record review. The study protocol was approved by the Institutional Review Board of The University of Texas M. D. Anderson Cancer Center.

\section{Metabolic profiling}

Metabolomics profiles were obtained using Liquid chromatography triple quadrupole mass spectrometry (LC-QQQ-MS) at Northwest Metabolomics Research Center, University of Washington. Briefly, LC-MS/MS was performed using an electrospray ionization source and the multiple-reaction-monitoring mode. A Sciex 5500 QTRAP triple quad MS system equipped with an Agilent 1200 ultra-high-pressure liquid chromatography system was utilized. The MS acquisition for each plasma sample $(0.050 \mathrm{~mL}$ each) will target a list of 224 metabolites. In addition, 24 isotope-labeled standards were included in each sample run for quality control and comparisons with other studies or additional samples run at a different time (e.g. discovery vs validation). The average $\mathrm{CV}$ of relative intensities for instrument QC samples was 5.3\% for the detected metabolites and the average $\mathrm{CV}$ of quantified metabolites using isotope-labeled internal standards was $3 \%$, indicating good reproducibility. The targeted 224 metabolites are selected from 25 key metabolic pathways, including alanine, aspartate, and glutamate metabolism; arginine and proline metabolism; butanoate metabolism; the citrate cycle (TCA cycle); cysteine and methionine metabolism; fatty acid metabolism; glutathione metabolism; glycine, serine, and threonine metabolism; glycolysis/gluconeogenesis; histidine metabolism; lysine biosynthesis; lysine degradation; nitrogen metabolism; oxidative phosphorylation; pentose phosphate pathway; phenylalanine metabolism; phenylalanine, tyrosine, and tryptophan biosynthesis; purine metabolism; pyrimidine metabolism; pyruvate metabolism; synthesis and degradation of ketone bodies; tryptophan metabolism; tyrosine metabolism; valine, leucine, and isoleucine biosynthesis; and valine, leucine, and isoleucine degradation. Certain metabolites are involved in multiple metabolic pathways. The raw data are processed using MultiQuant software (AB SCIEX) to integrate chromatographic peaks, and the data are visually inspected to ensure the quality of signal integration. The peak area will be calculated for each individual metabolite.

\section{Statistical analysis}

Any missing values were assumed to be below the limits of detection, and these values were imputed with the compound minimum (minimum value imputation). Statistical analysis of log-transformed data was conducted using MetaboAnalyst 3.0 http://www.metaboanalyst.ca/. Welch $t$ tests were conducted to compare data accounted for by estimating false discovery rate (FDR) using $q$ values. Logistic regression analysis was applied to assess the relationship between individual metabolite and tumor grade, GBM status, or IDH mutation status. Covariates, including age, gender, ethnicity, KPS, steroid use, seizure medication use, and dyslipidemia diagnosis, were adjusted in the analysis. Principal component analysis (PCA) and partial least squares-discriminant analysis (PLS-DA) were carried out using log-transformed data. Metabolic pathway analysis was conducted to identify the enriched metabolite sets and significant metabolic pathways that could differentiate glioma grade (high vs low), GBM versus malignant glioma, and $I D H$ mutation status (positive vs negative). The latest version of The Human Metabolome Database (HMDB) 3.0 was used in metabolic pathway analysis [35]. In addition, pathway topology analysis was used to estimate the impact of a certain metabolite or a group of metabolites in a certain metabolic pathway, and relative-betweeness centrality test was used to estimate the impact.

\section{CONFLICTS OF INTEREST}

None declared. 


\section{REFERENCES}

1. Ramakrishna R, Pisapia D. Recent Molecular Advances in Our Understanding of Glioma. Cureus. 2015; 7:e287.

2. Cohen AL, Colman H. Glioma biology and molecular markers. Cancer Treat Res. 2015; 163:15-30.

3. Cancer Genome Atlas Research N. Comprehensive genomic characterization defines human glioblastoma genes and core pathways. Nature. 2008; 455:1061-1068.

4. Cancer Genome Atlas Research N. Brat DJ, Verhaak RG, Aldape KD, Yung WK, Salama SR, Cooper LA, Rheinbay E, Miller CR, Vitucci M, Morozova O, Robertson AG, Noushmehr H, Laird PW, et al. Comprehensive, Integrative Genomic Analysis of Diffuse Lower-Grade Gliomas. N Engl J Med. 2015; 372:2481-2498.

5. Xu XD, Shao SX, Jiang HP, Cao YW, Wang YH, Yang XC, Wang YL, Wang XS, Niu HT. Warburg effect or reverse Warburg effect? A review of cancer metabolism. Oncol Res Treat. 2015; 38:117-122.

6. Chen X, Qian Y, Wu S. The Warburg effect: evolving interpretations of an established concept. Free Radic Biol Med. 2015; 79:253-263.

7. Chinnaiyan P, Kensicki E, Bloom G, Prabhu A, Sarcar B, Kahali S, Eschrich S, Qu X, Forsyth P, Gillies R. The metabolomic signature of malignant glioma reflects accelerated anabolic metabolism. Cancer Res. 2012; 72:5878-5888.

8. Griffin JL, Atherton H, Shockcor J, Atzori L. Metabolomics as a tool for cardiac research. Nat Rev Cardiol. 2011; 8:630-643.

9. Spratlin JL, Serkova NJ, Eckhardt SG. Clinical applications of metabolomics in oncology: a review. Clinical cancer research. 2009; 15:431-440.

10. Lloyd SM, Arnold J, Sreekumar A. Metabolomic profiling of hormone-dependent cancers: a bird's eye view. Trends Endocrinol Metab. 2015; 26:477-85.

11. Lucarelli G, Rutigliano M, Galleggiante V, Giglio A, Palazzo S, Ferro M, Simone C, Bettocchi C, Battaglia M, Ditonno P. Metabolomic profiling for the identification of novel diagnostic markers in prostate cancer. Expert Rev Mol Diagn. 2015; 15:1211-1224.

12. Mishra P, Ambs S. Metabolic Signatures of Human Breast Cancer. Mol Cell Oncol. 2015; 2.

13. Wojakowska A, Chekan M, Widlak P, Pietrowska M. Application of metabolomics in thyroid cancer research. Int J Endocrinol. 2015; 2015:258763.

14. Urayama S. Pancreatic cancer early detection: expanding higher-risk group with clinical and metabolomics parameters. World journal of gastroenterology. 2015; 21:1707-1717.

15. Derr RL, Ye $X$, Islas MU, Desideri S, Saudek CD, Grossman SA. Association between hyperglycemia and survival in patients with newly diagnosed glioblastoma. Journal of clinical oncology. 2009; 27:1082-1086.

16. Chambless LB, Parker SL, Hassam-Malani L, McGirt MJ, Thompson RC. Type 2 diabetes mellitus and obesity are independent risk factors for poor outcome in patients with high-grade glioma. Journal of neuro-oncology. 2012; 106:383-389.

17. Eckel-Passow JE, Lachance DH, Molinaro AM, Walsh KM, Decker PA, Sicotte H, Pekmezci M, Rice T, Kosel ML, Smirnov IV, Sarkar G, Caron AA, Kollmeyer TM, et al. Glioma Groups Based on 1p/19q, IDH, and TERT Promoter Mutations in Tumors. N Engl J Med. 2015; 372:2499-2508.

18. Kwon H, Oh S, Jin X, An YJ, Park S. Cancer metabolomics in basic science perspective. Archives of pharmacal research. 2015; 38:372-380.

19. Phillips MM, Sheaff MT, Szlosarek PW. Targeting argininedependent cancers with arginine-degrading enzymes: opportunities and challenges. Cancer Res Treat. 2013; 45:251-262.

20. Copeland RA, Moyer MP, Richon VM. Targeting genetic alterations in protein methyltransferases for personalized cancer therapeutics. Oncogene. 2013; 32:939-946.

21. Allen MD, Luong P, Hudson C, Leyton J, Delage B, Ghazaly E, Cutts R, Yuan M, Syed N, Lo Nigro C, Lattanzio L, Chmielewska-Kassassir M, Tomlinson I, et al. Prognostic and therapeutic impact of argininosuccinate synthetase 1 control in bladder cancer as monitored longitudinally by PET imaging. Cancer Res. 2014; 74:896-907.

22. Delage B, Fennell DA, Nicholson L, McNeish I, Lemoine NR, Crook T, Szlosarek PW. Arginine deprivation and argininosuccinate synthetase expression in the treatment of cancer. Int J Cancer. 2010; 126:2762-2772.

23. Pavlyk I, Rzhepetskyy Y, Jagielski AK, Drozak J, Wasik A, Pereverzieva G, Olchowik M, Kunz-Schugart LA, Stasyk O, Redowicz MJ. Arginine deprivation affects glioblastoma cell adhesion, invasiveness and actin cytoskeleton organization by impairment of beta-actin arginylation. Amino Acids. 2015; 47:199-212.

24. Katz J, Tayek JA. Recycling of glucose and determination of the Cori Cycle and gluconeogenesis. Am J Physiol. 1999; 277:E401-407.

25. Guo C, Pirozzi CJ, Lopez GY, Yan H. Isocitrate dehydrogenase mutations in gliomas: mechanisms, biomarkers and therapeutic target. Curr Opin Neurol. 2011; 24:648-652.

26. Lee SH, Suh JW, Chung BC, Kim SO. Polyamine profiles in the urine of patients with leukemia. Cancer Lett. 1998; 122:1-8.

27. Yerli H, Agildere AM, Ozen O, Geyik E, Atalay B, Elhan AH. Evaluation of cerebral glioma grade by using normal side creatine as an internal reference in multi-voxel 1H-MR spectroscopy. Diagn Interv Radiol. 2007; 13:3-9.

28. Sanson M, Marie Y, Paris S, Idbaih A, Laffaire J, Ducray F, El Hallani S, Boisselier B, Mokhtari K, Hoang-Xuan K, Delattre JY. Isocitrate dehydrogenase 1 codon 132 mutation is an important prognostic biomarker in gliomas. J Clin Oncol. 2009; 27:4150-4154.

29. Capper D, Simon M, Langhans CD, Okun JG, Tonn JC, Weller M, von Deimling A, Hartmann C, German Glioma N. 
2-Hydroxyglutarate concentration in serum from patients with gliomas does not correlate with IDH1/2 mutation status or tumor size. Int J Cancer. 2012; 131:766-768.

30. Agathocleous M, Harris WA. Metabolism in physiological cell proliferation and differentiation. Trends Cell Biol. 2013; 23:484-492.

31. Tam VC. Lipidomic profiling of bioactive lipids by mass spectrometry during microbial infections. Semin Immunol. $2013 ; 25: 240-248$.

32. Elkhattouti A, Hassan M, Gomez CR. Stromal Fibroblast in Age-Related Cancer: Role in Tumorigenesis and Potential as Novel Therapeutic Target. Front Oncol. 2015; 5:158.

33. Nakano I, Garnier D, Minata M, Rak J. Extracellular vesicles in the biology of brain tumour stem cells-Implications for inter-cellular communication, therapy and biomarker development. Semin Cell Dev Biol. 2015; 40:17-26.

34. Pavlides S, Tsirigos A, Migneco G, Whitaker-Menezes D, Chiavarina B, Flomenberg N, Frank PG, Casimiro MC, Wang C, Pestell RG, Martinez-Outschoorn UE, Howell A, Sotgia F, et al. The autophagic tumor stroma model of cancer: Role of oxidative stress and ketone production in fueling tumor cell metabolism. Cell Cycle. 2010; 9:3485-3505.

35. Wishart DS, Jewison T, Guo AC, Wilson M, Knox C, Liu Y, Djoumbou Y, Mandal R, Aziat F, Dong E, Bouatra S, Sinelnikov I, Arndt D, et al. HMDB 3.0-The Human Metabolome Database in 2013. Nucleic Acids Res. 2013; 41:D801-807. 